\title{
Multichannel Cognitive Cross Layer Optimization for Improved Video Transmission
}

\author{
Manimekalai Thirunavukkarasu, Meenakshi Murugappan and Maneesha S. Mohan
}

Department of Electronics and Communication Engineering,

Faculty of Information and Communication Engg, College of Engineering Guindy, Chennai, India

Received 2012-11-04, Revised 2013-01-09; Accepted 2013-02-06

\begin{abstract}
Multimedia applications particularly real-time video transmission in wireless networks, envisions end to end user perceived video quality as an important QoS parameter to be achieved. Cognitive Radio promising efficient spectrum utilization combined with Cross layer optimization is seen as a powerful combination to achieve the desired video quality. This study proposes Optimal Channel Sensed Multichannel Cognitive MAC (OCSMCMAC), a QoS driven cross layer system for the joint optimization of different network parameters along the network protocol stack for the improved video transmission. The primary network activity and wireless propagation dependent channel quality are modeled. Depending on the availability of the primary channel and channel condition as provided by an optimal sensing scheme and the encoder parameter in the application layer, cognitive MAC scheduling and PHY layer modulation and coding for the secondary user are optimized to achieve the required QoS. The simulation of channel and the cognitive user activity is done in MATLAB, while the application video coding is performed by H.264/AVC JM 15.1 codec to obtain the results. The results of the proposed OCSM-CMAC scheme demonstrate that improved PSNR and delay performance is achieved under the optimal channel sensing scheme compared to the random sensing scheme.
\end{abstract}

Keywords: Wireless Video Transmission, Cross Layer Design, Channel Sensing, Cognitive Network, Verage PSNR, End to End Delay

\section{INTRODUCTION}

Real-time video streaming and online video gaming are seen as important applications in today's wireless communication devices. These applications require large bandwidth and need to satisfy stringent QoS constraints. Cross-layer designs are seen as a suitable solution to improve QoS performance. Authors in (Oh and Chen, 2009) present a cross-layer design for a reliable video transmission over wireless ad hoc networks based on a multichannel MAC protocol with TDMA. Further, authors in (Setton et al., 2005; Delgado et al., 2006; Schaar and Shankar, 2005) propose different cross-layer based solutions for video-streaming over wireless ad hoc networks. With regard to the bandwidth requirement for real-time video streaming, the common belief is that the spectrum is scarce. Contrary to this, regulatory bodies in various countries (including the Federal Communications Commission in the United States and Ofcom in the United Kingdom) have found that most of the radio frequency spectrum is actually used inefficiently. This finding has stimulated a flurry of exciting activities in engineering, economics and regulatory communities in searching for better spectrum management policies and techniques (Zhao and Sadler, 2007). The basis for all these policies is that the licensed spectrum assigned to the primary users if underutilized can be exploited by the unlicensed or secondary users opportunistically. This calls for cognitive capability by the unlicensed users. Software defined and cognitive the unlicensed users. Software defined and Faculty of Information and Communication Engg, College of Engineering Guindy, Chennai, India 
radios provide a solution to this problem. Softwaredefined radio, is generally a multiband radio that supports multiple air interfaces and protocols and is reconfigurable through software run on FPGA, DSP or general-purpose microprocessors (Mitola, 2000). Cognitive radio, built on a software radio platform, is a context-aware intelligent radio potentially capable of autonomous reconfiguration by learning from and adapting to the communication environment. A survey of literature on real-time video streaming shows that there are only limited amount of work carried out for video transmission over cognitive networks using cross layer design. In this research work an attempt is made to improve the performance of real-time video transmission using cross-layer design and cognitive approach.

Cognitive Radio (CR), represents a broader paradigm where many aspects of communication systems can be improved via cognition. Dynamic spectrum access in CR is an important aspect with three basic components, namely spectrum identification, spectrum exploitation and spectrum regulation. Spectrum identification involves sensing the licensed primary user frequencies /channels for busy or idle condition, Spectrum exploitation involves efficient usage of idle frequencies/channels and spectrum regulation involves spectrum usage by secondary users maximizing their performance without degrading the performance of primary networks. The trade-off between the secondary users' desire for performance and the primary users' need for protection dictates effective interaction across opportunity identification, opportunity exploitation and regulatory policy. The optimal design of Opportunistic Spectrum Access (OSA) thus calls for a cross-layer approach that integrates signal processing and networking with regulatory policy making (Zhao and Sadler, 2007; Akildiz et al., 2006).

There are a number of works carried out in cross layer design for cognitive networks. The cross layer design challenges in spectrum management and spectrum handoff and challenges in higher layers are discussed in (Akildiz et al., 2006). Li et al. (2005) information such as link state information to enable adaptive modulation, the traffic flow in network layer to optimize congestion at transport layer, application layer coding and security are exchanged between different layers of the protocol stack. End-to-end performance is optimized by adapting to this information at each protocol layer. Unlike the primary wireless networks, in cognitive network the spectrum sensing and spectrum management in the presence of sensing errors is a challenging task. From the literature it is known that there are numerous research work done in spectrum sensing. Authors in ( $\mathrm{Hu}$ et al., 2010) focus on the fairness among the multicast users in CR networks that have infrastructure based $C R$ network base stations with multiple transceivers that sense multiple channels simultaneously. Authors in (Shiang and Schaar, 2008) suggest a dynamic channel selection scheme based on the priority packet scheduling for multiuser CR networks but they do not concentrate on channel sensing. A survey of challenges in spectrum sensing and the trade-offs involved are given by Ghasemi (2008). There are numerous sensing algorithms proposed in the literature. Su and Zhang (2008) proposed two sensing algorithms namely Negotiation based Sensing Policy (NSP) and Random Sensing Policy (RSP) which make use of co operation among the secondary users in a distributed network. In NSP, secondary users improve channel sensing efficiency by negotiation with each other and thereby performance is optimized. In RSP a kind of AND logic is used in making a decision about the primary channel activity which is a more conservative approach. Jia et al. (2008) HC-MAC protocol that represents the sensing process as an optimal stopping problem in order to determine how long a cognitive radio should observe the wireless bands to optimize its expected throughput is discussed. Here channel bonding and aggregation techniques set the limit for the finite horizon problem.

In this work, the cross layer based cognitive MAC proposed by Luo et al. (2011), is extended to multichannel CMAC and optimized by considering the optimal channel selection done based on simple channel sensing order proposed by Tung and Zhuang (2011) for CR networks. The system model considered in this study has no sufficient knowledge about the primary network activity and hence the energy detection based sensing is considered. Further, the optimal sensing time (Liang et al., 2008) for energy detection is considered with which the highest throughput for the secondary network is achieved, while the primary users are protected. Each secondary user in the current work is considered to have only one transceiver and employs a unicast video transmission, unlike the multicast case proposed by $\mathrm{Hu}$ et al. (2010). The proposed Optimal Channel Sensed Multichannel Cognitive MAC (OCSM-CMAC) is shown to result in significant reduction in waiting time of a packet thereby maximizing the average PSNR and minimizing end to end delay for improved video transmission over the cognitive network.

The major contributions in this work are as follows: 
- The OCSM-CMAC is proposed wherein, optimal channel sensing scheme is applied in a cross layer based cognitive network, which coexists with the multichannel primary network, to support high quality video transmission between the secondary users

- QoS driven cross layer system for the joint optimization of different network parameters across the network protocol stack is proposed. The activity of the primary network and wireless channel is modelled. Depending on the availability of the primary channel and the channel condition provided by the optimal sensing scheme and the encoder parameter in the application layer, parameters like cognitive MAC scheduling, PHY layer modulation and coding are optimized for the secondary user to achieve the required QoS

- The impact of increase in the number of available primary channels and thereby the probability of getting a free primary channel with good channel state are analysed and shown that significant reduction in the scheduling delay and the transmission time can be achieved leading to an improved performance

- Finally the impact of combining the cross layer optimization with optimal channel sensing scheme is analysed and demonstrated to outperform the cross layer optimization with a random sensing scheme

\subsection{System Model}

In this study, a CR network with one non-mobile secondary user pair and $\mathrm{N}$ available primary channels is considered. The proposed OCSM-CMAC cross-layer optimized system includes different modules, such as video encoder module, multichannel cognitive MAC module, cognitive PHY module, cross-layer optimization module, as well as wireless video transmission module. The cross-layer optimization module is the core of the proposed system. The multichannel cognitive MAC module resides in the MAC layer, while the cognitive PHY module is in the PHY layer. As shown in Fig. 1 the cross-layer optimization module communicates with other modules to adjust the network functions, by selecting the optimal system parameters so as to maximize the average PSNR of the received video with reduced delay. In this way, the network functions are jointly optimized to achieve the best user-perceived video quality over CR networks under different network conditions.

The objective function in the proposed optimization is the Expected Video Distortion calculated at the video encoder of the application layer (Regunathan et al., 2001) as the PSNR value of the received video frame is an indirect method of measuring distortion caused. It can be represented by the encoding parameter such as quantization step size $q$ and packet loss rate $\rho$. The expected packet delay, which is jointly represented by system parameters that affect encoder behaviour, channel selection, MAC scheduling, transmission and modulation and channel coding, is used as the design constraint. Thus the cross layer optimization module selects the optimum set of parameters in order to achieve improved user perceived video quality.

\subsection{Cross Layer Optimization Parameters}

\subsubsection{Distortion Calculation}

At the video encoder of the application layer, all possible factors need to be considered, to accurately estimate the video distortion that could be caused at the decoder. For the source coding parameter, Quantization step size (QP) q is considered in this study.

Given the dependencies introduced by the error concealment scheme, the expected distortion of packet $\mathrm{x}$ of video frame $\mathrm{g}$ can be calculated (Regunathan et al., 2001) as Equation 1:

$$
\begin{aligned}
& E\left[D_{g, x}\right]=\left(1-\rho_{x}\right) E\left[D^{r}{ }_{g, x}\right]+\rho_{x}\left(1-\rho_{x-1}\right) E\left[D_{g, x}^{\operatorname{lr}}\right] \\
& +\rho_{x} \rho_{x-1} E\left[D^{11}{ }_{g, x}\right]
\end{aligned}
$$

where, $\rho_{x}$ is the end-to-end loss probability of packet $x$ with consideration of packet delay bound. $E\left[D_{g, x}^{r}\right]$ is the expected distortion of packet $\mathrm{x}$ if received, while $\mathrm{E}\left[\mathrm{D}_{\mathrm{g}, \mathrm{x}}^{\mathrm{lr}}\right]$ and $\mathrm{E}\left[\mathrm{D}_{\mathrm{g}, \mathrm{x}} \mathrm{ll}\right]$ are the expected distortions of the lost packet $\mathrm{x}$ after concealment if packet $(\mathrm{x}-1)$ is received or lost, respectively.

Therefore, the expected distortion of the whole video frame $g$ can be represented as (Luo et al., 2011) Equation 2:

$\mathrm{E}\left[\mathrm{D}_{\mathrm{g}}\right]=\sum_{\mathrm{x}=1}^{\mathrm{x}_{\mathrm{g}}} \mathrm{E}\left[\mathrm{D}_{\mathrm{g}, \mathrm{x}}\right]$

where, $X_{\mathrm{g}}$ is the total number of packets in video frame $\mathrm{g}$.

\subsection{Channel Model and Optimal Sensing}

In this study, $\mathrm{N}$ licensed wireless channels are considered and they are assumed to be frequency flat and the primary users activities are assumed to be time synchronised. As the secondary user pair is considered to be non-mobile, the channel gain of their transmission link is considered to be time-invariant. 


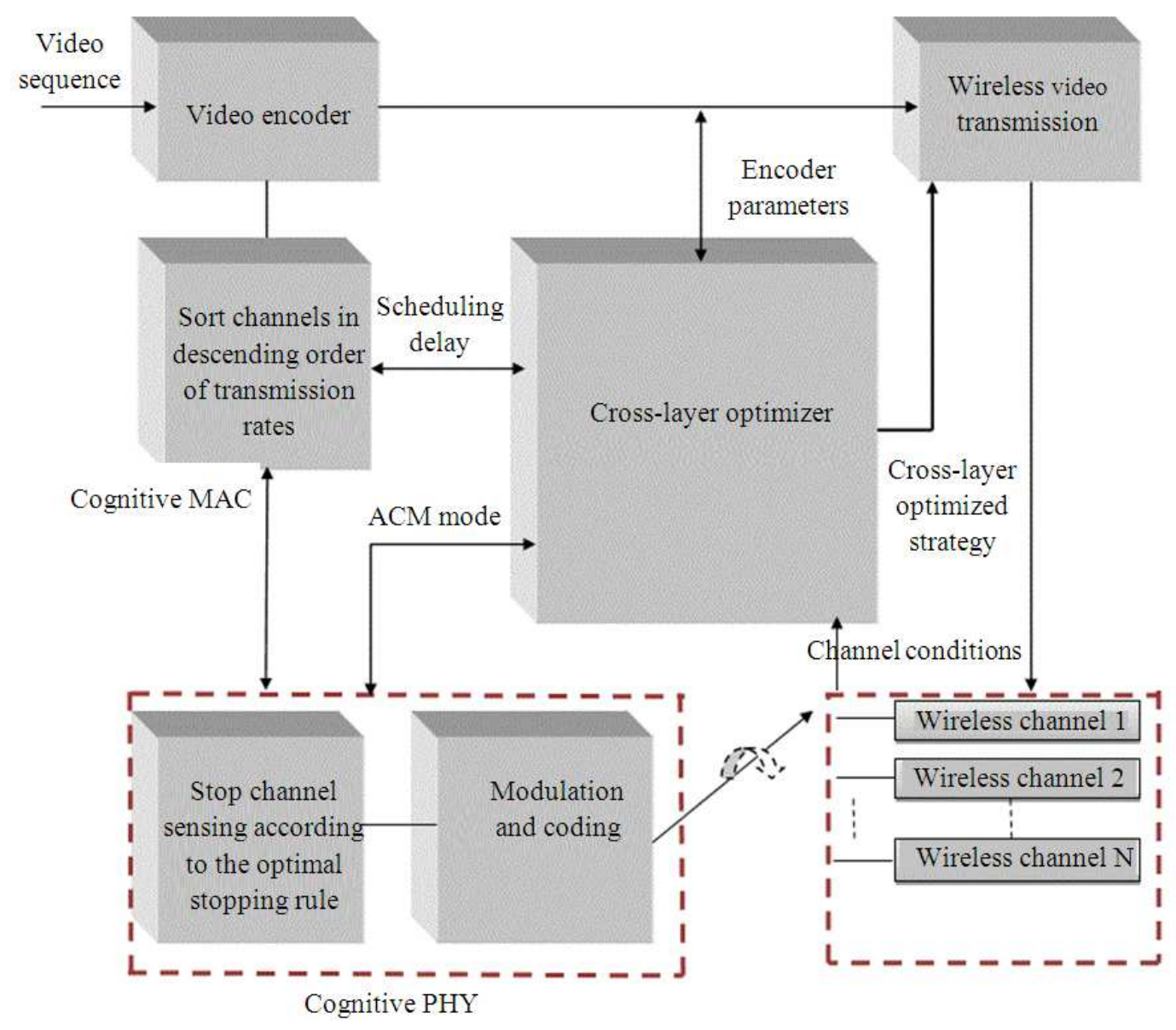

Fig. 1. Proposed OCSM-CMAC cross-layer system model for wireless video transmission in CR networks

The primary channel quality is measured by the received Signal-to-Noise Ratio (SNR) $\eta$. The Rayleigh channel model is used to describe $\eta$ statistically. Therefore, the received SNR is a random variable with a probability density function as follows Equation 3:

$$
p(\eta)=(1 / \eta ́) \exp (-\eta / \eta ́), \eta \geq 0
$$

where, $\dot{\eta}=\mathrm{E}\{\eta\}$ is the average received SNR (Holm, 2002; Luo et al., 2011). It is also assumed that the secondary receiver has perfect channel state information as in (Tung and Zhuang, 2011).

The secondary users in the system model considered do not have sufficient knowledge about the primary network activity and hence the energy detection based channel sensing is considered. For Optimal sensing in a system with a single pair of non-mobile secondary users and $\mathrm{N}$ available primary channels, time is partitioned into slots of duration $\mathrm{T}_{\mathrm{o}}$. In each time-slot, each channel is either available (no primary activity) or busy (primary active) namely ON or OFF respectively. The availability of a channel is considered i.i.d. in both frequency channel and time-slot dimensions, (Su and Zhang, 2008). The channels are sensed by the source node of a secondary user pair according to a sensing sequence. Denote $\left(\mathrm{s}_{1}, \mathrm{~s}_{2}, \ldots, \mathrm{s}_{\mathrm{N}}\right)$ as a sensing sequence, which is a permutation of the set $(1,2, \ldots, N)$, (Fan and Jiang, 2009). Denote $\tau$ as the time needed for sensing a channel, where $\mathrm{N} \tau<<\mathrm{T}_{\mathrm{o}}$. The channels are sensed by energy detection method and each sensing attempt is independent of previous attempts. As each node is equipped with only a single transceiver, the secondary users can detect only one channel at a time. Figure 2 depicts the slot structure and channel exploitation under consideration, where the source node of a secondary user pair senses the first (k-1) channels as busy and stops at the $\mathrm{k}^{\text {th }}$ sensed channel if the optimal stopping criterion which is discussed in section $\mathrm{C}$ is satisfied. The secondary user then transmits its 
information over the $\mathrm{k}^{\text {th }}$ sensed channel in the reminder of that timeslot. The period of time spent on channel sensing and that on data transmission are $\mathrm{k} \tau$ and $\left(\mathrm{T}_{\mathrm{o}}-\mathrm{k} \tau\right)$, respectively. With a constant slot time, the actual data transmission time changes with $\mathrm{k}$. Let $\mathrm{C}_{\mathrm{k}}$ be the time duration available for data transmission in a time slot, if a secondary user pair stops at the $\mathrm{k}^{\text {th }}$ sensed channel, it is given by Equation 4:

$$
\mathrm{C}_{\mathrm{k}}=\left(\mathrm{T}_{\mathrm{o}}-\mathrm{k} \tau\right)
$$

In other words, the larger the value of $\mathrm{k}$, the smaller the value of $C_{k}$ and the lower the effective data transmission. A secondary user transmits only when the channel is sensed free. The SNR estimation of the sensed free channel and the well known Shannon capacity formula are used to calculate the transmission rate supported by the sensed channel.

\subsection{Optimal Stopping Rule and Channel Exploitation}

Channel exploitation in $\mathrm{CR}$ networks should effectively and efficiently utilize an idle spectrum/channel. In fact, the problem of channel exploitation can be viewed as a general stopping rule problem, the objective of which is to stop at the channel that maximizes the (expected) reward for a secondary user pair. In this study, with a finite number of channels, the channel exploitation problem can be formulated as a finite-horizon stopping problem, (Ferguson, 2004).

A secondary user pair is assumed to need only one channel to transmit its data. Since the transmission rates of all the channels can be known via channel estimation and considering larger coherence time, the source node of a secondary user pair senses the channels according to the descending order of their achievable rates. Therefore, for $\mathrm{i}<\mathrm{j}, \mathrm{R}_{\mathrm{si}} \geq \mathrm{R}_{\mathrm{sj}}, \forall \mathrm{i}, \mathrm{j}$, where $\mathrm{R}_{\mathrm{sk}}$ is the achievable transmission rate of the $\mathrm{k}^{\text {th }}$ sensed channel with the secondary user pair using full transmit power. If the $\mathrm{s}_{\mathrm{k}}^{\text {th }}$ channel is sensed free, a secondary user pair can stop at the $\mathrm{s}_{\mathrm{k}}^{\text {th }}$ channel and transmit its data at the rate of $\mathrm{R}_{\mathrm{sk}}$; otherwise, it proceeds to sense the $s_{k+1}$ th channel.

Stopping Rule -If a secondary user pair needs only one channel for data transmission, it should stop sensing at the first free channel. With the use of channel sensing order, it can be proved that the secondary user pair achieves the maximal (expected) reward by stopping at the first sensed free channel and, hence, the stopping rule is optimal (Tung and Zhuang, 2011).

The cognitive channel model used in this study has the secondary users trying to transmit data when primary users are not present and active. Secondary users will first perform optimal channel sensing to detect the activity of primary users in the $\mathrm{N}$ available channels and then decide whether to transmit the data immediately or wait for a free channel in the next time slot depending on the detection result.

Following are the four states of a given primary channel:

State $\mathrm{s}_{1}$ : Channel is idle, detected as idle (correct detection)

State $s_{2}$ : Channel is idle, detected as busy (false alarm)

State $\mathrm{s}_{3}$ : Channel is busy, detected as idle (missed detection)

State $\mathrm{s}_{4}$ : Channel is busy, detected as busy (correct detection)

Here channel sensing problem is formulated as a binary hypothesis testing problem. Therefore, the two hypotheses defined based on the noisy observation over $\mathrm{M}$ symbol periods are Equation 5:

$$
\begin{array}{ll}
y_{i}=n_{i}: H_{0} & i=1, \ldots, M \\
y_{i}=h \cdot s_{i}+n_{i}: H_{1} & i=1, \ldots, M
\end{array}
$$

where, $h$ is ' 0 ' for $H_{0}$ and ' 1 ' for $H_{1}$ and the received noise $\left\{\mathrm{n}_{\mathrm{i}}\right\}$ is a zero mean, complex Gaussian random variable with variance ${\sigma_{\mathrm{n}}}^{2}$ for all $\mathrm{i}$, denoted as $\left\{\mathrm{n}_{\mathrm{i}}\right\} \sim$ $\mathrm{M}\left(0, \sigma_{\mathrm{n}}{ }^{2}\right) .\left\{\mathrm{s}_{\mathrm{i}}\right\}$ is the sum of the active primary users' faded signals arriving at the secondary receiver, which has a circularly symmetric complex Gaussian distribution with zero-mean and variance $\sigma_{\mathrm{s}}^{2}$. Both $\left\{\mathrm{s}_{\mathrm{i}}\right\}$ and $\left\{\mathrm{n}_{\mathrm{i}}\right\}$ are assumed to be independent and identically distributed. Therefore, as per Neyman-Pearson algorithm the detection problem becomes Equation 6:

$$
\mathrm{Y}=\frac{1}{\mathrm{M}} \sum_{\mathrm{i}=1}^{\mathrm{M}}\left|\mathrm{y}_{\mathrm{i}}\right|_{<\mathrm{H}_{1}}^{>\mathrm{H}_{0}} \mathrm{t}_{\mathrm{d}}
$$

where, $t_{d}$ is the detection threshold. The test statistic $Y$ is chi-square distributed with $2 \mathrm{M}$ degrees of freedom. Therefore, the probabilities of detection $p_{d}$ and false alarm $p_{\mathrm{f}}$ can be represented as follows Equation 7 and 8:

$$
\begin{aligned}
& \mathrm{p}_{\mathrm{d}}=\mathrm{P}\left(\mathrm{Y}>\mathrm{t}_{\mathrm{d}} \mid \mathrm{H}_{1}\right) \\
& \mathrm{p}_{\mathrm{f}}=\mathrm{P}\left(\mathrm{Y}>\mathrm{t}_{\mathrm{d}} \mid \mathrm{H}_{0}\right)
\end{aligned}
$$

where, $p_{d}$ is the probability of detecting the channel correctly and $\mathrm{p}_{\mathrm{f}}$ is the probability of false alarm. 


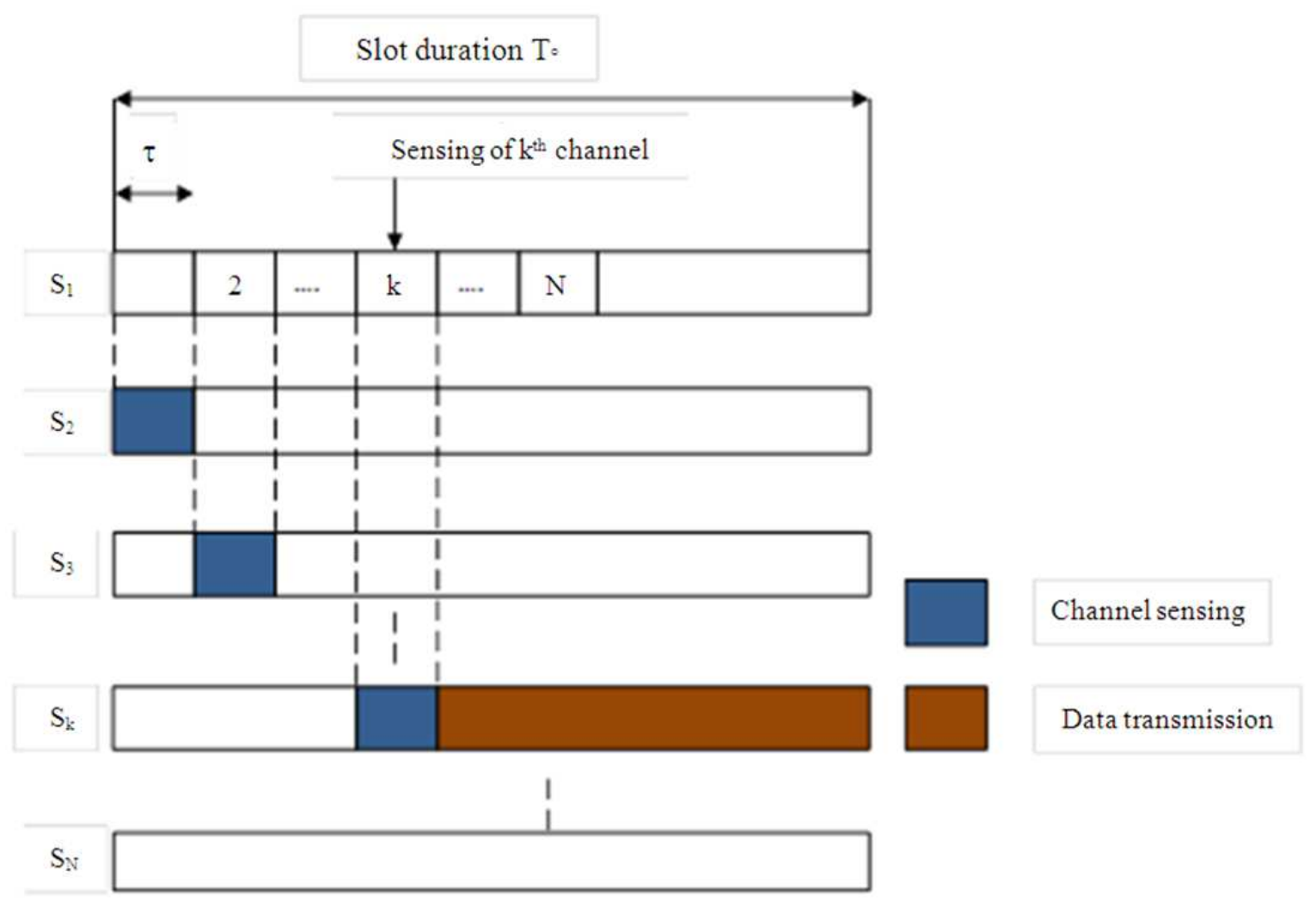

Fig. 2. Channel sensing procedure for a secondary user

The probability of a channel being idle and detected as idle can therefore be calculated as Equation 9:

$\mathrm{p}_{11}=\left(1-\mathrm{p}_{\mathrm{f}}\right)\left(1-\xi_{\mathrm{b}}\right)$

where, $\xi_{\mathrm{b}}$ is the prior probability of channel being busy. This indicates that the transition probability only depends on the current state, regardless of the original state. The remaining transition probabilities can also be obtained in terms of $\mathrm{p}_{\mathrm{d}}, \mathrm{p}_{\mathrm{f}}$ and $\xi_{\mathrm{b}}$.

\subsection{MAC Scheduling Delay}

To formulate the MAC frame scheduling delay for secondary users in CR networks, $T_{0}$ is fixed as the duration of a time slot and $\tau$ the corresponding channel sensing time allocated for each of the channels in a time slot such that $\mathrm{N} \tau<\mathrm{T}_{0}$, as shown in Fig. 2. As per OCSM-CMAC the secondary user will stop sensing once it finds the first free channel (in state $s_{1}$ ) and that channel can support maximum data rate. If all of the available channels sensed are in state $s_{2}$ or $s_{4}$, the MAC frame has to wait for the next time slot. In the next time slot, if all of the sensed channels are still in state $s_{2}$ or $s_{4}$, it has to wait again. This process repeats until one of the channels sensed in a time slot becomes available, or until a maximum waiting threshold denoted as $\mathrm{N}_{\text {max }}^{\mathrm{s}}$ in terms of the number of time slots is reached. If this maximum threshold is reached, the waiting packet has to be dropped from the sending queue. Furthermore, to ensure real-time video transmission, every video packet has to meet a delay bound. Therefore, all the video packets of the same video frame have the same delay bound. $\mathrm{T}_{\mathrm{g}}^{\max }$ is denoted as the delay bound of the video frame $\mathrm{g}$. Then, $\mathrm{N}_{\text {max }}^{\mathrm{s}}$ can be calculated as, (Luo et al., 2011) Equation 10:

$\mathrm{N}_{\max }^{\mathrm{s}}=\left\lfloor\frac{\mathrm{T}_{\mathrm{g}}^{\max }}{\mathrm{T}_{0}}\right\rfloor$

The $\mathrm{N}$ channels are sensed in the descending order of transmission rates. As shown in Fig. 3, only when a channel is in state $s_{1}$, it can be effectively used to transmit data for secondary users. When the channel is in state $s_{2}, s_{3}$, or $s_{4}$, it is not available for secondary users, or it is not detected by the secondary users as available. 


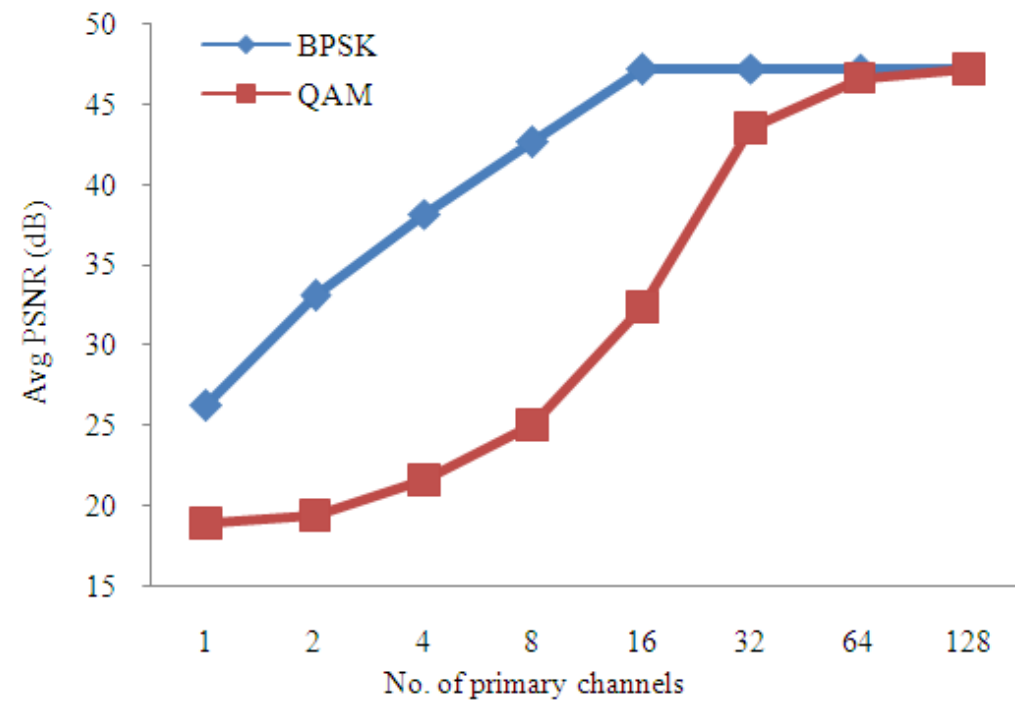

Fig. 3. Average PSNR for varying number of available primary channel without cross layer at physical layer i.e., with fixed modulation

Therefore, the probability $\mathrm{p}_{\text {wait }}$ that a given MAC frame has to wait for the next time slot in the $\mathrm{N}$ channel MAC (OSCM-CMAC) can be expressed as Equation 11:

$$
\mathrm{p}_{\text {wait }}=\prod_{\mathrm{i}=1}^{\mathrm{N}}\left[1-\mathrm{p}_{\mathrm{f}_{\mathrm{i}}}\left(1-\xi_{\mathrm{b}_{\mathrm{i}}}\right)\right]
$$

Here, $p_{f_{i}}$ is the probability of false alarm for the $i^{\text {th }}$ channel and $\xi_{\mathrm{bi}}$ is the a priori probability of the $\mathrm{i}^{\text {th }}$ channel being sensed busy. The given MAC frame has to wait for the next time slot only when all the $\mathrm{N}$ channels are sensed busy. Hence, the probability $p_{w a i t}$ is the product of the probabilities of the $\mathrm{N}$ channels being sensed busy.

Thus, assuming that the availability of time slots are independent, the average scheduling time can be represented as Equation 12:

$$
\begin{aligned}
& \mathrm{t}_{\text {sched }}=\left(\mathrm{p}_{\text {wait }}+\mathrm{p}_{\text {wait }}^{2}+\ldots+\mathrm{p}_{\text {wait }}^{\mathrm{N}_{\text {max }}^{\mathrm{s}}}\right) * \mathrm{~T}_{\mathrm{o}} \\
& \mathrm{t}_{\text {sched }}=\left(\frac{\mathrm{p}_{\text {wait }}-\mathrm{p}_{\text {wait }}^{\mathrm{N}_{\max }^{\mathrm{s}}}}{1-\mathrm{p}_{\text {wait }}}\right) * \mathrm{~T}_{\mathrm{o}}
\end{aligned}
$$

\subsection{Transmission Delay}

In this study, Adaptive Modulation and Coding (AMC) technique at the PHY layer is adopted on a packet-by-packet basis to enhance the throughput. With AMC, the optimal combination of different modulation constellations and different rates of error- control codes is selected based on the sensed channel quality. For example, if the sensed channel has good channel conditions, AMC schemes with larger constellation sizes and higher channel coding rate will guarantee the required packet error rate for quality of service provisioning. Usually, Bit Error Rate (BER), $\in$ ( $\eta$ ) can be calculated from the approximated expression as follows Equation 13:

$\in(\eta)=a_{m} e^{-b_{m} \eta}$

where, coefficients $a_{m}$ and $b_{m}$ and be obtained by fitting this equation to the exact BER. Therefore, the frame error rate $\Phi(\eta)$ can be expressed as Equation 14:

$\Phi(\eta)=1-(1-\in(\eta))^{1}$

where, 1 is the variable MAC frame size and $\eta$ is the received SNR of the sensed channel.

Denote B as the bandwidth (symbols/s) and $1_{x}(\mathrm{~m})$, the number of bits in packet $x$ with mode $m$. The transmission delay can then be represented as, (Luo et al., 2011) Equation 15:

$$
\mathrm{t}_{\text {trans }}=\left\lceil\frac{\frac{\mathrm{l}_{\mathrm{x}}(\mathrm{m})}{\mathrm{r}_{\mathrm{m}} \mathrm{B}}}{\mathrm{T}_{\mathrm{o}}-\mathrm{k} \tau}\right\rceil * \mathrm{~T}_{\mathrm{o}}
$$

where, $r_{m}$ is the rate (bits/symbol) of AMC mode $\mathrm{m}$ as shown in Table 1. 
Table 1. AMC schemes at cognitive PHY

\begin{tabular}{llllll}
\hline AMC mode $(\mathrm{m})$ & $\mathrm{m}=1$ & $\mathrm{~m}=2$ & $\mathrm{~m}=3$ & $\mathrm{~m}=4$ & $\mathrm{~m}=5$ \\
\hline Modulation scheme & BPSK & QPSK & QPSK & $16-\mathrm{QAM}$ & $16-\mathrm{QAM}$ \\
Coding rate $\left(\mathrm{c}_{\mathrm{m}}\right)$ & $1 / 2$ & $1 / 2$ & $3 / 4$ & $9 / 16$ & $3 / 4$ \\
$\mathrm{r}_{\mathrm{m}}$ & 0.50 & 1.00 & 1.50 & 2.25 & 3.00 \\
\hline
\end{tabular}

\subsection{Problem Formulation}

To achieve the best video quality at the receiver side, the expected end-to-end video distortion E [D], under the constraint of video packet delay should be minimized. In the proposed distortion-delay framework, the source coding, MAC scheduling, transmission and modulation and coding are jointly optimized in a cross-layer fashion. In wireless video transmission, all packets of a given frame $g$ is constrained by a frame delay bound $\mathrm{T}_{\mathrm{g}}^{\max }$ and all packets of the video frame $g$ have the same delay constraint $\mathrm{T}_{\mathrm{g}}^{\max }$. Denote $\mathrm{Q}$ as the set of all possible operating points of source coding parameter (such as quantization step size $\mathrm{q}_{\mathrm{g}, \mathrm{x}}$ ) of packet $\mathrm{x}$ of frame $\mathrm{g}$, $\mathrm{M}$ as set of all possible modulation and channel coding schemes $\mathrm{m}_{\mathrm{g}, \mathrm{x}}$. Thus, the proposed problem can be formulated as a minimization of average distortion, given by Equation 16:

$$
\begin{gathered}
\left\{\min _{\left.\mathrm{g}, \mathrm{x} \in \mathrm{Q}, \mathrm{m}_{\mathrm{g}, \mathrm{x}} \in \mathrm{M}\right\}} \sum_{\mathrm{g}=1}^{\mathrm{G}} \sum_{\mathrm{x}=1}^{\mathrm{X}_{\mathrm{g}}} \mathrm{E}\left[\mathrm{D}_{\mathrm{g}, \mathrm{x}}\right]\right. \\
\text { Subject to: } \max _{1 \leq \mathrm{x} \leq \mathrm{X}_{\mathrm{g}}} \mathrm{t}_{\mathrm{g}, \mathrm{x}} \leq \mathrm{T}_{\mathrm{g}}^{\max }, \forall \mathrm{x}
\end{gathered}
$$

where, $G$ is the total number of video frames of the given video sequence and $X_{g}$ is the total number of packets generated from the $\mathrm{g}^{\text {th }}$ video frame. Therefore, the proposed problem has been formulated into a MIN-MAX problem, (Li et al., 2005).

\section{MATERIALS AND METHODS}

In this study, video coding is performed by H.264/AVC JM 15.1 codec. The video sequence Foreman is adopted for performance analysis. 10 frames of the video sequence are coded at $25 \mathrm{f} / \mathrm{s}$ with each I frame being followed by nine $\mathrm{P}$ frames. When a packet is lost during transmission, the temporal-replacement error concealment strategy is applied. The motion vector of a missing Macro Block can be estimated as the median of motion vectors of the nearest three MBs in the preceding row. The pixels in the previous frame, pointed by the estimated motion vector, are used to replace the missing pixels in the current frame.
In the experiments, QP $\mathrm{q}$ and AMC mode $\mathrm{m}$ of each packet are considered as the parameters to be optimized. The encoder is set to operate under the Rate Distortion Optimized mode to adjust the rate and hence the quantization parameter in an inherent manner. The available AMC schemes are indexed 1 to 5 as shown in Table 1. The generator polynomial of the mother code is $\mathrm{g}=[133 ; 171]$. The coding rates are obtained from the puncturing pattern P2 of the HIPERLAN/2 standard, (Liu et al., 2004). Depending on the channel status one among the five modes will be chosen. The threshold SNR values for switching between the different modes are fixed such that a BER of $10^{-4}-10^{-6}$ is satisfied as per the requirements for video transmission. The main focus of this work is to ensure the improved video reception performance for secondary users with cross layer optimization in association with idle channel selection based on optimal sensing order, where sensing is assumed to be reliable with negligible probability of false alarm. The wireless channel is modeled in a simulator. Given an average SNR $\eta$, the instantaneous link quality $\eta$ can be randomly produced. In this study, the link bandwidth is set to $100 \mathrm{Ksymbols} / \mathrm{s}$ under a single hop scenario. The delay bound is set to get optimum performance. For video applications, Peak Signal-to-Noise Ratio (PSNR) and Average end-to-end delay of the received video frames of secondary users are used as the performance metrics to compare the proposed system having multichannel cognitive MAC with the system having a single channel CMAC (Luo et al., 2011) and also to compare the performance of the optimal sensing approach in the current scenario with random sensing approach.

\section{RESULTS AND DISCUSSION}

The performance of the proposed Optimal Channel Sensed Multichannel Cognitive MAC (OCSM-CMAC) is first studied for partial cross layer optimization i.e., for fixed modulation and coding. By varying the number of available primary channels, the results are obtained for average PNSR and Average end-to-end delay per frame, as shown in Fig. 3 and 4 respectively, with packet delay bound of $10 \mathrm{~m} \mathrm{sec}$ and time slot duration of $5 \mathrm{~m} \mathrm{sec}$.

When the constellation size is small PSNR value is good but the delay is excessive which could be an issue in video application. At the same time when the constellation size is increased, as expected, there is significant reduction in delay but the PSNR performance is poor. This necessitates the cross layer communication between MAC and PHY layers to adjust the modulation and coding depending on the link status. 
Manimekalai Thirunavukkarasu et al. / Journal of Computer Science 9 (1) (2013) 43-54

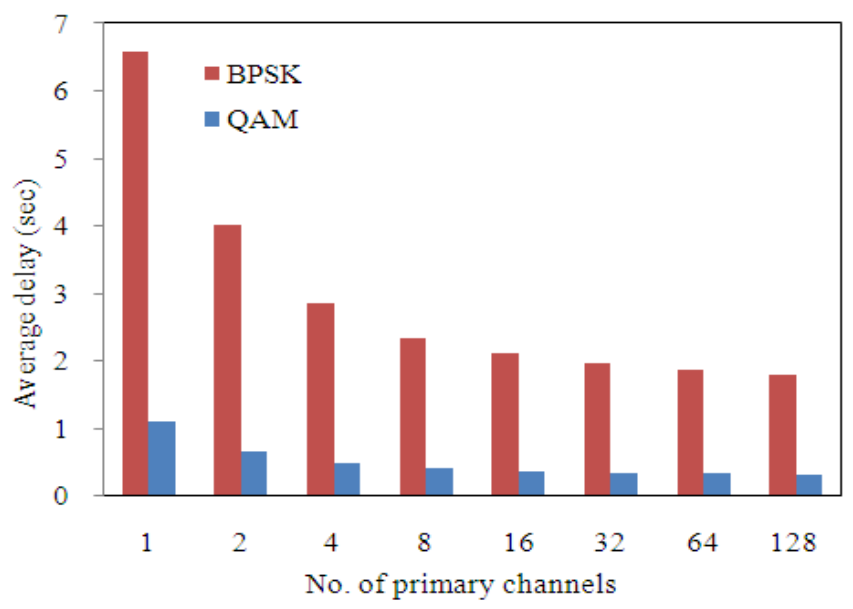

Fig. 4. Average delay for varying number of available primary channel, without cross layer at physical layer i.e., with fixed modulation

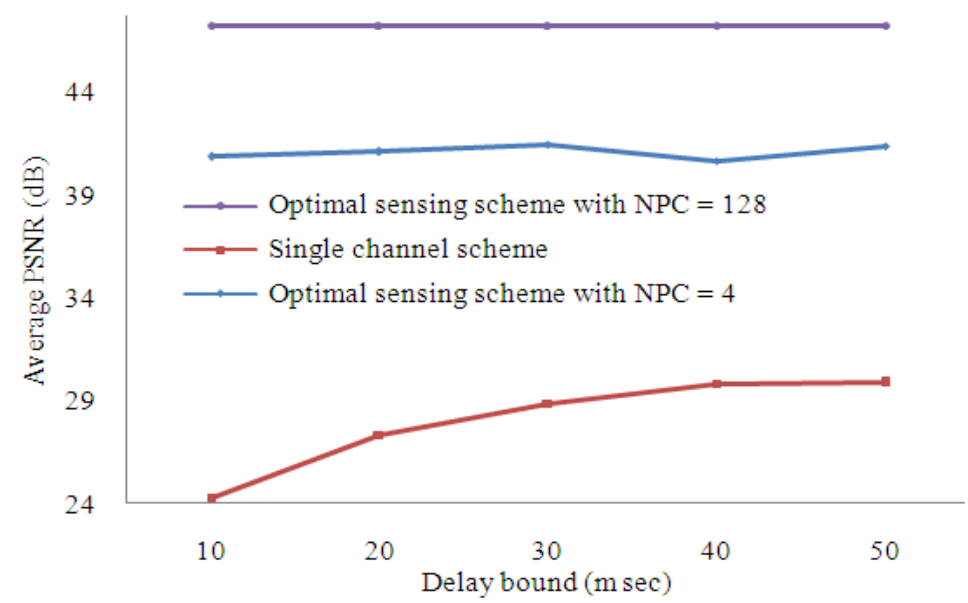

Fig. 5. Average PSNR per frame versus delay bound per packet for time slot duration of $5 \mathrm{~m} \mathrm{sec}$

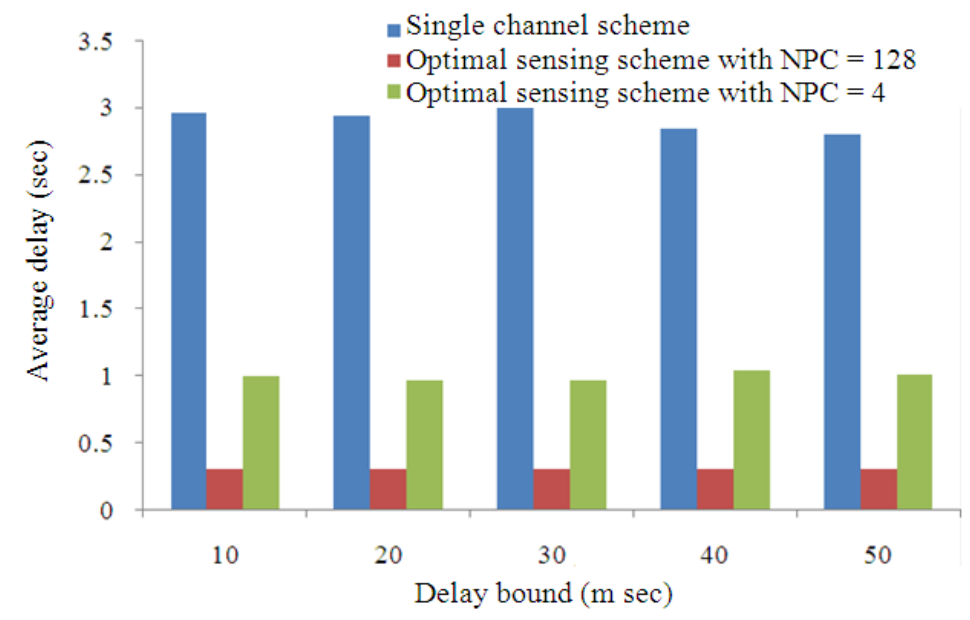

Fig. 6. Average end to end delay frame versus delay bound per packet for time slot duration of $5 \mathrm{~m} \mathrm{sec}$ 


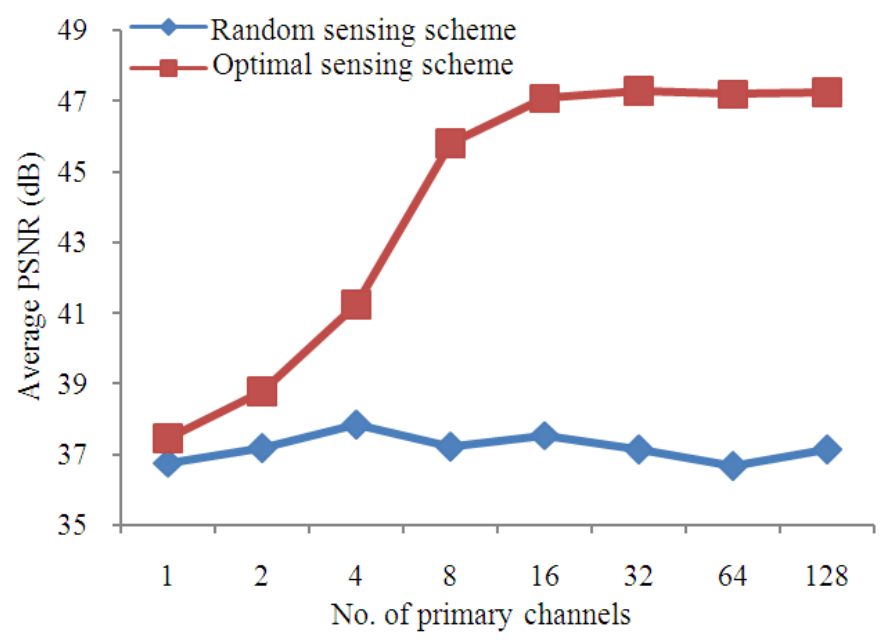

Fig. 7. Average PSNR for varying number of available primary channel with cross layer optimization for Delay Bound $=30 \mathrm{~m} \mathrm{sec}$

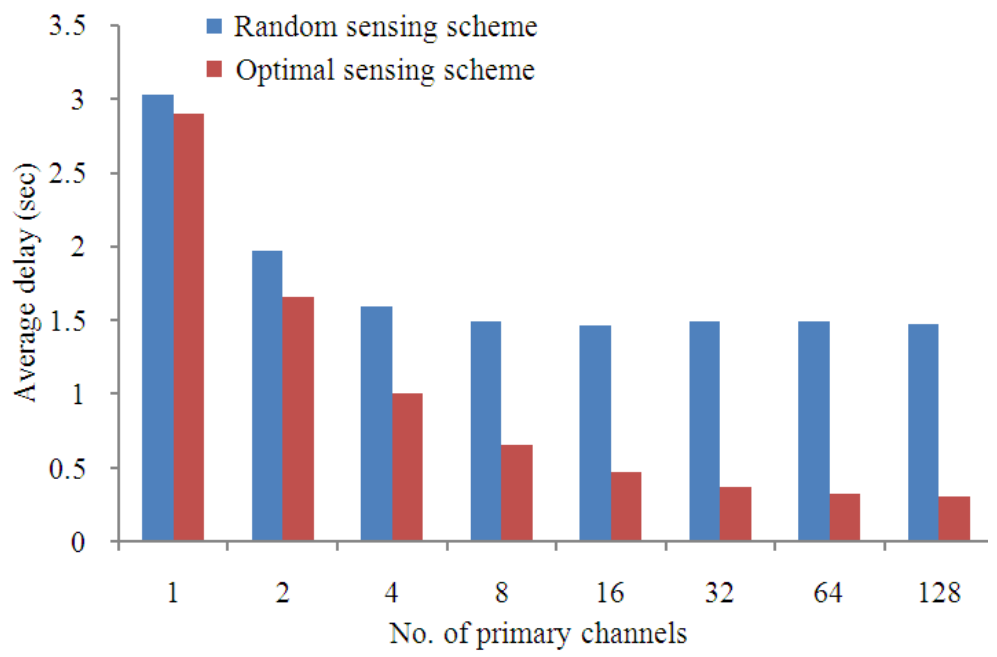

Fig. 8. Average end to end delay per frame for varying number of primary channels with delay bound of $30 \mathrm{~ms}$ and time slot duration of $1 \mathrm{~m} \mathrm{sec}$

In the case of a single channel Cognitive MAC, the PSNR is a function of the allowable packet delay bound. As there is only a single channel to be sensed, when the delay bound is set at a minimum value, the number of time slots for which a secondary user can wait to sense and find the single channel to be free gets reduced. Once the delay bound is exceeded without finding the single channel to be free, the packet is dropped leading to distortion. The same is observed in the results plotted in Fig. 5. As the delay bound becomes higher the PSNR increases. In the case of the proposed OCSM-CMAC, since there is more Number of Primary Channels (NPC) the waiting time of the packet is negligible and thereby the probability of the packet being dropped is expected to decrease, improving the PSNR. These observations are confirmed by the simulation results shown in Fig. 5, for $\mathrm{NPC}=4$ and $\mathrm{NPC}=128$. Also, higher the NPC, higher is the PSNR, as expected. Additionally, it is also observed that under the multichannel scenario, the PSNR performance is independent of the delay bound and depends only on the NPCs.

The delay performance of the single channel CMAC and the proposed OCSM-CMAC are compared in Fig. 6. The proposed OCSM-CMAC again shows a better 
performance as expected. Since there are multiple channels and the channels sensed as free are also ordered according to their link quality, there is always a free channel with better link state that is available at the earliest which can be exploited with higher AMC mode thereby ensuring significant reduction in end to end delay. Here again the performance is seen to be independent of the delay bound and depends only on the NPCs.

The simulations were then carried out to compare the performance of multichannel cognitive cross layer optimization for Random sensing and optimal sensing. The average PSNR and average delay results obtained and shown in Fig. 7 and 8 respectively, indicate that the average PSNR per frame is high for optimal sensing with cross layer design compared to random sensing and the increase in PSNR as the number of primary channels are increased is due to the fact that when the number of channels are more there is a higher probability of sensing free channels with good SNR status.

In Fig. 8, the average end to end delay per frame is also observed to be significantly less for optimal sensing with increase in number of primary channels, because of the higher mode of the AMC selected under good channel condition and the increased transmission rate. In Random sensing, though there could be channels with better SNR available, the reduction in delay is less significant because the channels are not ordered based on their SNR and the first free channel is selected for transmission, irrespective of its link state.

The impact of the Time Slot duration is then studied for both Random sensing based Multichannel CMAC and the proposed OCSM-CMAC and observed that the results are independent of variation in time slot duration.

\section{CONCLUSION}

The Optimal Channel Sensed Multichannel Cognitive MAC (OCSM-CMAC) is proposed in this study for realtime video transmission in wireless networks, with end to end user perceived video quality as an important QoS parameter to be satisfied. Cognitive Radio for efficient spectrum utilization is combined with Cross layer optimization to achieve the desired video quality. The results obtained show a significant reduction in waiting time of a packet due to the incorporation of Optimal sensing, thereby maximizing the average PSNR and minimizing end to end delay for improved video transmission over the cognitive network. The proposed technique is also observed to show a performance that is independent of the delay bound and time slot duration when there are more number of primary channels. This work shows the performance improvement for video transmission in $\mathrm{CR}$ network with optimal channel sensing order considering single pair of nodes with no contention. The impact on the performance for multiple pair of nodes on a contention basis is the scope for further study and analysis.

\section{REFERENCES}

Akildiz, I.F., W.Y. Lee, M.C.Vuran and S. Mohanty, 2006. NeXt generation/dynamic spectrum access/cognitive radio wireless networks: A survey. Commun. Netw., 50: 2127-2159. DOI: 10.1016/j.comnet.2006.05.001

Delgado, G.D., V.C. Frias and M.A. Igartua, 2006. ViStA-XL: A cross-layer design for video-streaming over ad hoc networks. ISWCS.

Fan, R. and H. Jiang, 2009. Channel sensing-order setting in cognitive radio networks: A two-user case. IEEE Trans. Veh. Technol., 58: 4997-5008. DOI: 10.1109/TVT.2009.2027712

Ferguson, S.T., 2004. Optimal stopping and application.

Ghasemi, A., 2008. Spectrum sensing in cognitive radio networks: Requirements, challenges and design trade-offs. IEEE Commun. Mag., 46: 32-39. DOI: 10.1109/MCOM.2008.4481338

Holm, H., 2002. Adaptive coded modulation performance and channel estimation tools for flat fading channels. Ph.D. dissertation in partial fulfillment of the requirements for the degree of Doctor of Philosophy, Department of Telecommunications Norwegian University of Science and Technology.

Hu, D., S. Mao, Y.T. Huo and J.H. Reed, 2010. Scalable video multicast in cognitive radio networks. IEEE J. Selected Areas Commun., 28: 334-344. DOI: 10.1109/JSAC.2010.100414

Jia, J., Q. Zhang and X. Shen, 2008. HC-MAC: A hardware-constrained cognitive MAC for efficient spectrum management. IEEE J. Selected Areas Commun., 26: 106-117. DOI: 10.1109/JSAC.2008.080110

Li, Z., M. Guido S. Aggelos and K. Katsaggelos, 2005. Minmax optimal video summarization. IEEE Trans. Circ. Syst. Video Technol., 15: 1245-1256. DOI: 10.1109/TCSVT.2005.854230

Liang, Y.C., Y. Edward, C.Y. Peh and A.T. Hoang, 2008. Sensing-throughput tradeoff for cognitive radio networks. IEEE Trans. Wireless Commun., 7: 1326-1337. DOI: 10.1109/TWC.2008.060869 
Liu, Q., S. Zhou and B.G. Giannakis, 2004. Cross-Layer combining of adaptive modulation and coding with truncated ARQ over wireless links. IEEE Trans. Wireless Commun., 3: 1746-1755. DOI: 10.1109/TWC.2004.833474

Luo, H., S. Ci and D. Wu, 2011. A cross-layer design for the performance improvement of real-time video transmission of secondary users over cognitive radio networks. IEEE Trans. Circ. Syst. Video Technol., 21: 1040-1048.

DOI: 10.1109/TCSVT.2011.2129810

Mitola, J., 2000. Software Radios: Wireless Architecture for the 21st Century. 1st Edn., Wiley, NewYork.

Oh, B.J. and C.W. Chen, 2009. A cross-layer approach to multichannel MAC protocol design for video streaming over wireless ad hoc networks. IEEE Trans. Multimedia, 11: 1052-1061. DOI: 10.1109/TMM.2009.2026083

Regunathan, S., R. Zhang and K. Rose, 2001. Scalable video coding with robust mode selection. Signal Proc. Image Commun., 16: 725-732. DOI: 10.1016/S0923-5965(01)00003-0

Schaar, M.V.D. and S.N. Shankar, 2005. Cross-layer wireless multimedia transmission: Challenges, principles and new paradigms. IEEE Wireless Commun., 12: 50-58. DOI: 10.1109/MWC.2005.1497858
Setton, E., T. Yoo, X. Zhu, A. Goldsmith and B. Girod, 2005. Cross-layer design of ad hoc networks for Real-time video streaming. IEEE Wireless Commun. Mag., 12: 59-65. DOI: 10.1109/MWC.2005.1497859

Shiang, H.P. and M.V.D. Schaar, 2008. Dynamic channel selection for multi-user video streaming over cognitive radio networks. Proceedings of the International Conference, on Image Processing, Oct. 12-15, IEEE Xplore Press, San Diego, CA., pp: 2316-2319. DOI: 10.1109/ICIP.2008.4712255

Su, H. and X. Zhang, 2008. Cross-layer based opportunistic mac protocols for qos provisionings over cognitive radio wireless networks. IEEE J. Selected Areas Commun., 26: 118-129. DOI: 10.1109/JSAC.2008.080111

Tung, H.C. and W. Zhuang, 2011. Simple channel sensing order in cognitive radio networks. IEEE J. Selected Areas Commun., 29: 676-688. DOI: 10.1109/JSAC.2011.110402

Zhao, Q. and B.M. Sadler, 2007. A survey of dynamic spectrum access. IEEE Signal Proc. Mage., 24: 7989. DOI: 10.1109/MSP.2007.361604 